\title{
Interpretation of Regressions with Multiple Proxies*
}

\author{
Darren Lubotsky \\ Research Program in Development Studies \\ Princeton University \\ Princeton, NJ 08544 \\ lubotsky@princeton.edu
}

\author{
Martin Wittenberg \\ Economics Department \\ University of the Witwatersrand \\ South Africa \\ 060wim@mentor.edcm.wits.ac.za
}

August 2001

\begin{abstract}
We consider the situation in which there are multiple proxies for one unobserved explanatory variable in a linear regression and provide a procedure by which the coefficient of interest can be extracted "post hoc" from a multiple regression in which all the proxies are used simultaneously. This post hoc estimator is strictly superior in large samples to coefficients derived using any index or linear combination of the proxies that is created prior to the regression. To use an index created from the proxies that extracts the largest possible signal from them requires knowledge of information that is not available to the researcher. Using the proxies simultaneously in a multiple regression delivers this information. and the researcher then simply combines the coefficients in a known way to obtain the estimate of the effect of the unobserved factor. This procedure is also much more robust than ad hoc index construction to departures from the assumption of an underlying common factor. We provide some Monte Carlo simulations and applications to existing empirical problems to show that the reduction in attenuation bias can be non-negligible. even in finite samples.
\end{abstract}

JEL C'lassification: C1. C31

Keywords: Proxy variables, measurement error. index construction.

\footnotetext{
"We thank Anne Case. Angus Deaton. Bo Honorć. Hashem Pesaran. Elic Taner and seminar participants at the African Econometrics Conference and Princeton University for their comments and suggestions. We are naturally responsible for any crrors. This work began while Martin Wittenberg was visiting the Research Program in Development Studies at Princeton University. Martin Wittenberg acknowledges financial support from Investec Bank through their urant to Econometric Research Southern Africa. and we both acknowledge financial support from the John D. and (atherine T. MacArthur Foundation through their Network on Poverty and Inequality in a Broader Perspective.
} 


\section{Introduction}

There are many contexts in which economists encounter badly measured variables or are forced to use proxies of one form or another. While a great deal of attention has been paid to effects of a single mismeasured independent variable in a regression model, much less is known about the analytics and empirical strategies when more than one measure or proxy of the independent variable is available. Instead, researchers often rely on improvised or arbitrary methods. Consider the following strategies culled from recent studies:

\section{The effect of social capital and bad governance on economic performance}

People's willingness to engage in civic activities and trust those with whom they interact may lower the cost of exchange and facilitate cooperative solutions, and therefore increase the economic performance of organizations or whole economies. Bureaucratic and judicial corruption and inefficiency, and political instability may raise the costs of exchange and harm growth. Unfortunately, these concepts are not easily measured and researchers must rely on proxy variables. For example, trust is commonly measured by responses to the following questions in the General Social Survey: "Generally speaking, would you say that most people can be trusted or that you can't be too careful in dealing with people?" "Do you think most people would try to take advantage of you if they got the chance, or would they try to be fair?" and "Would you say that most of the time people try to be helpful, or that they are mostly just looking out for themselves?"

How would one best use these variables to test for an underlying relationship between trust and economic performance or cooperative behavior? One option would be to enter the measures one at a time as explanatory variables and see whether any of them have significant predictive power, though this risks the perception that the researcher picked certain results to fit a particular story. Another option is to construct an index of the various proxy measures that might simultaneously utilize all of the information contained in the three questions. The difficulty here is that if the questions contain different amounts of information, how should they be aggregated?

Glaeser et al (2000) create an index of trust by standardizing (subtracting the mean 
and dividing by the standard deviation) the above variables and then adding them up. Mauro (1995) uses indices of political and labor stability, "red tape", corruption, terrorism, and several other outcomes compiled by Business International, a private consulting firm, to measure institutional efficiency and corruption. Since he believes many of these indices measure the same underlying phenomena, he averages the indices together and uses the average as a regressor in models of growth and investment across countries.

\section{Permanent income and intergenerational mobility}

Estimating the effect of parents' permanent income on the education, health, and subsequent earnings of their children has a long history in the social sciences. Permanent income is not observed; instead, observed income in any year includes transitory components representing luck, measurement error, and other unanticipated shocks to income. If parents' investment in their children is a function of permanent income, then the correlation between observed income and children's outcomes will understate the true effect of permanent income on outcomes. A standard approach in the literature has been to average parents' income over several years as a measure of permanent income. See, for example, Solon (1992) and Zimmerman (1992).

Parent's income is not available at all in the British National Child Development Survey, an otherwise rich, 30-plus year follow-up survey of nearly every child born in Britain during the week of March 3, 1958. Instead, data is only available for each parents' education and social class, a seven-point scale based on occupation. One might think of alternative ways to use both the education and social class data to best capture parents' economic status.

\section{Wealth effects when income and wealth are not observed}

The Demographic and Health Surveys are large household datasets with nearly identical questionnaires in over 40 developing counties. Unfortunately, no information is available on income, wealth, or consumption. Filmer and Pritchett (2001) propose using the first principal component from 21 measures of ownership of consumer durables (a car or television, for example), dwelling characteristics (source of drinking water, toilet 
facilities), and landownership to construct an "asset index," which they then use to estimate the effect of household resources on children's educational enrollment. The first principal component is the linear combination of the asset variables that accounts for the highest proportion of their variance. Subsequent principal components are orthogonal linear combinations that explain the highest proportion of the remaining variation. Filmer and Pritchett (2001) suggest that a natural interpretation of the first component is household wealth.

In many of these cases the authors have chosen to summarize the proxies in a single, new variable. There are several considerations underlying this strategy. Firstly by taking some linear combination of the proxies, the measurement error problem may be reduced. As Mauro (1995) notes:

Part of the rationale for aggregating the indices into composite subindices is that there may be measurement error in each individual index, and averaging the individual indices may yield a better estimate of the determinants of investment and growth.

Secondly, we may be better able to interpret the output. Indeed if the unobserved variable is not of intrinsic interest, but is merely a nuisance variable to be controlled, then many researchers would not hesitate to simply include all the available proxies in an attempt to absorb its effect, even if they might not be able to interpret an individual coefficient (see for example Bertrand and Mullainathan 2001). If, however, the unobservable is the key variable of interest then it is not clear how to extract the "wealth" effect on education, for example, from the reported effects of owning a television or a radio.

Thirdly, researchers may be worried about multicollinearity. If the different proxies are in fact all measuring the same underlying phenomenon, then there are no separate coefficients to be estimated. This will be reflected in many insignificant individual coefficients.

We will show that all of these concerns are incorrect, but incorrect in interesting ways. Attenuation bias is in fact maximally reduced when all the proxies are used separately in a multiple regression. Furthermore there is a way in which the underlying 
coefficient can be extracted after the event. And the fact that the proxies all measure the same phenomenon does not detract from our ability to estimate that coefficient. In addition, our procedure has the virtue of being more transparent - its validity is based on the null hypothesis that there really is a common latent variable. If this hypothesis is not true, then it does not invalidate the regression, only some of the inference that is drawn from it. By contrast data manipulations done before the regression can obviously not be undone by a sceptical reader.

The plan of our discussion is as follows. In the following section we will introduce the basic problem we wish to investigate and the related literature. The main theoretical results are in section 3 . We provide some simulation evidence in section 4 and then turn to a discussion of a number of particular examples in section 5 . We conclude by pointing to a number of open questions, and an appendix contains the proofs of our main results.

\section{The basic problem}

The circumstances that we wish to investigate can be highlighted by means of the following equations:

$$
\begin{aligned}
y_{i} & =\beta x_{i}+\varepsilon_{i} \\
x_{1 i} & =x_{i}+u_{1 i} \\
x_{2 i} & =\rho_{2} x_{i}+u_{2 i}
\end{aligned}
$$

where $\beta$, relating $y_{i}$ and $x_{i}$ in equation $1 \mathrm{a}$, is the primary parameter of interest. We assume that $x_{i}$ is unobserved, but that we have the two proxies $x_{1 i}$ and $x_{2 i}$. Furthermore we will make the assumption that $u_{1 i}$ and $u_{2 i}$ are independent of $x_{i}$ and $\varepsilon_{i}$; that is, the proxy variables do not have an independent effect on $y_{i}$.

If we regress $y$ on the first proxy, we have the well-known case of classical measurement error with the attendant attenuation bias. The OLS estimator $b$ of $\beta$ will converge asymptotically to

$$
b=\beta \frac{\sigma_{x}^{2}}{\sigma_{x}^{2}+\sigma_{1}^{2}}
$$

where $\sigma_{x}^{2}=\operatorname{var}(x)$ and $\sigma_{1}^{2}=\operatorname{var}\left(u_{1}\right)$. The parameter $\beta$ is not identified. As Aigner, Hsiao, Kapteyn and Wansbeek (1984) note, we may be able to identify $\beta$ from higher 
order moments if the latent variable $x$ is not normally distributed.

In order to identify the parameter in general we need one more restriction. If we set $\operatorname{cov}\left(u_{1}, u_{2}\right)=0$, then we could use $x_{2}$ as an instrument for $x_{1}$ in the regression of $y$ on

$x_{1}$. A different type of restriction is if we are able to measure $\sigma_{1}^{2}$. This is possible, for instance, if the second "proxy" variable is actually a repeat measurement, carried out for a sub-set of observations under controlled conditions. In this case we can obviously also retrieve $\sigma_{x}^{2}$ and then correct the OLS estimates. This is the "errors in variables" estimator (see Fuller 1987).

We might consider whether the relationships between the proxies allow us to identify the common "factor" $x$. This is the domain of factor analysis only with even more stringent assumptions. Not only do we need to impose orthogonality between the error variances $u_{1}$ and $u_{2}$, but we also need to adopt a normalization on the coefficients. The "factors" so isolated are only identified up to multiplication by an orthogonal matrix.

Principal components analysis achieves a unique decomposition, but does so by the expedient of identifying the common factor with the linear combination of proxies that maximizes the combined variance. It is not clear why this concept should correspond to the structural relationships underlying equations 1a-1c. Indeed, if the assumption of orthogonality between the error variances fails, then this procedure is guaranteed to produce a composite of the factor $x$ and the commonality in the variances.

Other identification strategies involve adding equations or specifying the process which generates the latent variable. In the MIMIC (multiple indicators, multiple causes) model, for example (see inter alia Aigner et al. 1984, Goldberger 1972, Jöreskog and Goldberger 1975), it is assumed that there is at least one more relationship available between an indicator variable and the latent variable, parallel to that in equation 1a. The latent variable itself is written as a function of a series of observable variables, i.e. equations $1 \mathrm{~b}$ and $1 \mathrm{c}$ are replaced by

$$
x_{i}=\alpha_{1} x_{1 i}+\alpha_{2} x_{2 i}+u_{3}
$$

and the proportionality relationships between the different equations are exploited to achieve identification. We assume that these sorts of strategies are not available for the cases under consideration.

In particular, we assume that the researcher is not willing to make additional as- 
sumptions beyond those already given for equations 1a through $1 \mathrm{c}$, and the empirical problem is how to best use the proxy variables to minimize the attenuation bias, if not eliminate it. The issue therefore is how to make the best of a bad situation. Throughout, however, we restrict attention to models that are linear in the parameters.

Leamer (1983, pp.314-315) has a discussion of "proxy searches" in which he addresses precisely this issue. His discussion is, however, exclusively about how to decide which one of the two (or more) proxies to include in the regression. His advice is to pick the variable which yields a high $R^{2}$ and which has a low variance. He does not consider whether one could do better by combining the information from the proxies.

In order to hone our intuition, let us consider the system in equations 1a-1c with $\rho_{2}=1$. Let us assume that the covariance matrix of $x_{1}$ and $x_{2}$ is given by

$$
\Sigma_{X X}=\left[\begin{array}{cc}
\sigma_{x}^{2}+\sigma_{1}^{2} & \sigma_{x}^{2}+\sigma_{12} \\
\sigma_{x}^{2}+\sigma_{12} & \sigma_{x}^{2}+\sigma_{2}^{2}
\end{array}\right]
$$

where

$$
\Sigma_{U U}=\left[\begin{array}{cc}
\sigma_{1}^{2} & \sigma_{12} \\
\sigma_{12} & \sigma_{2}^{2}
\end{array}\right]
$$

is the covariance matrix of $u_{1}$ and $u_{2}$ with $\sigma_{12} \neq 0$. By our assumptions $\operatorname{cov}\left(y_{1}, x_{1}\right)=$ $\operatorname{cov}\left(y_{1}, x_{2}\right)=\beta \sigma_{x}^{2}$ and hence the coefficients estimated by from regressing $y$ on proxy 1 or proxy 2 are given asymptotically respectively by

$$
b^{1}=\beta \frac{\sigma_{x}^{2}}{\sigma_{x}^{2}+\sigma_{1}^{2}} \text { and } b^{2}=\beta \frac{\sigma_{x}^{2}}{\sigma_{x}^{2}+\sigma_{2}^{2}}
$$

Since the denominator is just the variance of the proxy variable, it is clear that the proxy with the smaller variance will give the better results.

What were to happen if we were to take a simple average of the two proxies? In this case

$$
\bar{x}_{i}=x_{i}+\bar{u}_{i}
$$

with $\operatorname{var}\left(\bar{u}_{i}\right)=\frac{1}{4}\left(\sigma_{1}^{2}+\sigma_{2}^{2}+2 \sigma_{12}\right)$. There clearly is no necessity that this be smaller than the minimum of $\sigma_{1}^{2}$ and $\sigma_{2}^{2}$. In particular, if one proxy is a good one and the other much worse, simply averaging them out is unlikely to be the optimal strategy. Other linear combinations of the variables are likely to get a much better reduction 
in the error variance. Indeed let $u_{0}=\delta_{1} u_{1}+\delta_{2} u_{2}$ be a linear combination such that $\delta_{1}+\delta_{2}=1$, then it is straightforward to show that the choice of $\delta_{1}$ that will minimize the variance of the weighted average is given by $\delta_{1}=\frac{\sigma_{2}^{2}-\sigma_{12}}{\sigma_{1}^{2}-2 \sigma_{12}+\sigma_{2}^{2}}$. The variance in this case will be $\frac{\sigma_{2}^{2} \sigma_{1}^{2}-\sigma_{12}^{2}}{\sigma_{1}^{2}-2 \sigma_{12}+\sigma_{2}^{2}}$, so that the estimate of $\beta$ with the minimum attenuation bias is given asymptotically by

$$
b^{*}=\beta \frac{\sigma_{x}^{2}}{\sigma_{x}^{2}+\frac{\sigma_{2}^{2} \sigma_{1}^{2}-\sigma_{12}^{2}}{\sigma_{1}^{2}-2 \sigma_{12}+\sigma_{2}^{2}}}
$$

Unfortunately, we do not know the variances and covariance of $u_{1}$ and $u_{2}$, and thus cannot compute this optimally weighted average of the proxies.

What happens if we run the regression of $y$ on both proxies? The multiple regression coefficients will be given asymptotically by $\Sigma_{X X}^{-1} \Sigma_{X y}$ where

$$
\Sigma_{X y}=\left[\begin{array}{l}
\beta \sigma_{x}^{2} \\
\beta \sigma_{x}^{2}
\end{array}\right]
$$

It is straightforward to check that

$$
\begin{aligned}
& b_{1}=\beta \frac{\sigma_{x}^{2}\left(\sigma_{2}^{2}-\sigma_{12}\right)}{\sigma_{x}^{2} \sigma_{1}^{2}+\sigma_{x}^{2} \sigma_{2}^{2}-2 \sigma_{x}^{2} \sigma_{12}+\sigma_{1}^{2} \sigma_{2}^{2}-\sigma_{12}^{2}} \\
& b_{2}=\beta \frac{\sigma_{x}^{2}\left(\sigma_{1}^{2}-\sigma_{12}\right)}{\sigma_{x}^{2} \sigma_{1}^{2}+\sigma_{x}^{2} \sigma_{2}^{2}-2 \sigma_{x}^{2} \sigma_{12}+\sigma_{1}^{2} \sigma_{2}^{2}-\sigma_{12}^{2}}
\end{aligned}
$$

This does not look very promising, but note that

$$
\begin{aligned}
b_{1}+b_{2} & =\beta \frac{\sigma_{x}^{2}\left(\sigma_{1}^{2}+\sigma_{2}^{2}-2 \sigma_{12}\right)}{\sigma_{x}^{2} \sigma_{1}^{2}+\sigma_{x}^{2} \sigma_{2}^{2}-2 \sigma_{x}^{2} \sigma_{12}+\sigma_{1}^{2} \sigma_{2}^{2}-\sigma_{12}^{2}} \\
& =b^{*}
\end{aligned}
$$

so that adding up the coefficients of the two variables yields (asymptotically) an estimate that is precisely equal to the optimal weighting of the proxies. What is even more remarkable is that we did not need to know anything about the relative magnitudes of error variances and covariances in order to achieve this result - the regression accomplished this by itself.

We will show in the next section that this result holds true more generally - that the attenuation bias is always smallest when all the proxies are used in a multiple regression. We need to proceed with some care, however, particularly in the situation where $\rho_{2} \neq 1$. In that case we note that a simple average of the variables is

$$
\bar{x}_{i}=\bar{\rho} x_{i}+\bar{u}_{i}
$$


with $\bar{\rho}=\frac{1+\rho_{2}}{2}$. To avoid this unknown rescaling of the latent variable $x$, we will generally want to take a weighted sum of the individual coefficients from the multiple regression.

\section{The general case}

We now assume that we have $k$ proxies, with

$$
x_{j}=\rho_{j} x+u_{j}
$$

We assume that $\operatorname{cov}\left(u_{j}, \varepsilon_{j}\right)=0, \operatorname{cov}\left(u_{j}, x_{h}\right)=0$ for all $j, h$ but that the covariance matrix of the error terms is unrestricted, i.e.

$$
\Sigma_{U U}=E\left(U^{\prime} U\right)=\left[\begin{array}{cccc}
\sigma_{1}^{2} & \sigma_{12} & \cdots & \sigma_{1 k} \\
\sigma_{12} & \sigma_{2}^{2} & \cdots & \sigma_{2 k} \\
\vdots & \vdots & \ddots & \vdots \\
\sigma_{1 k} & \sigma_{2 k} & \cdots & \sigma_{k}^{2}
\end{array}\right]
$$

where $U=\left[\begin{array}{llll}u_{1} & u_{2} & \ldots & u_{k}\end{array}\right]$.

\subsection{Identification}

We note that as it stands the $\rho_{j}$ terms are not identified. Multiplying all $\rho$ s by a constant would result in the same observations. Consequently we adopt the normalization (already used in equation $1 \mathrm{~b}$ ) that $\rho_{1}=1$. This amounts to fixing the scale of the latent variable $x$ in terms of the observable $x_{1}$.

The information that is available is contained in the covariance matrix

$$
\Sigma_{Z Z}=E\left(Z^{\prime} Z\right)=\left[\begin{array}{ccccc}
\beta^{2} \sigma_{x}^{2}+\sigma_{\varepsilon}^{2} & \beta \sigma_{x}^{2} & \beta \rho_{2} \sigma_{x}^{2} & \cdots & \beta \rho_{k} \sigma_{x}^{2} \\
\beta \sigma_{x}^{2} & \sigma_{x}^{2}+\sigma_{1}^{2} & \rho_{2} \sigma_{x}^{2}+\sigma_{12} & \cdots & \rho_{k} \sigma_{x}^{2}+\sigma_{1 k} \\
\beta \rho_{2} \sigma_{x}^{2} & \rho_{2} \sigma_{x}^{2}+\sigma_{12} & \rho_{2}^{2} \sigma_{x}^{2}+\sigma_{2}^{2} & \cdots & \rho_{2} \rho_{k} \sigma_{x}^{2}+\sigma_{2 k} \\
\vdots & \vdots & \vdots & \ddots & \vdots \\
\beta \rho_{k} \sigma_{x}^{2} & \rho_{k} \sigma_{x}^{2}+\sigma_{1 k} & \rho_{2} \rho_{k} \sigma_{x}^{2}+\sigma_{2 k} & \cdots & \rho_{k}^{2} \sigma_{x}^{2}+\sigma_{k}^{2}
\end{array}\right]
$$

where $Z=\left[\begin{array}{lllll}y & x_{1} & x_{2} & \ldots & x_{k}\end{array}\right]$.

There are altogether $\frac{k(k+1)}{2}$ unknown parameters in $\Sigma_{U U}, k-1$ parameters in $\rho$ and the parameters $\beta, \sigma_{x}^{2}$ and $\sigma_{\varepsilon}^{2}$, i.e. there are altogether $\frac{(k+1)(k+2)}{2}+1$ unknown parameters in $\Sigma_{Z Z}$, but only $\frac{(k+1)(k+2)}{2}$ pieces of observable information. We are therefore short of one restriction in order to identify the parameter $\beta$. 
Observe, however, that the vector $\rho$ is identified from the covariances between the dependent variable $y$ and the proxies:

$$
\rho_{j}=\frac{\operatorname{cov}\left(y, x_{j}\right)}{\operatorname{cov}\left(y, x_{1}\right)}
$$

As noted above there are several ways in which we could achieve identification of $\beta$ : any restriction on the covariance matrix of $X=\left[\begin{array}{llll}x_{1} & x_{2} & \ldots & x_{k}\end{array}\right]$ will do so in principle. Zero restrictions on any of $\sigma_{1 j}$ would allow us to use $x_{j}$ as an instrument for $x_{1}$. More generally, a zero restriction on $\sigma_{j h}$ would allow us to use $x_{h}$ as an instrument for $x_{j}$, but the resulting estimate would need to be rescaled to take account of the fact that $x_{j}$ is not on the same scale as the latent variable $x$. Since we have an estimator for $\rho_{j}$ this is easily achieved. We have

$$
\beta=\frac{\operatorname{cov}\left(y, x_{h}\right)}{\operatorname{cov}\left(x_{j}, x_{h}\right)} \frac{\operatorname{cov}\left(y, x_{j}\right)}{\operatorname{cov}\left(y, x_{1}\right)}
$$

The first term is the "instrumental variables" estimator while the second is the GMM estimator of $\rho_{j}$.

If we know the magnitudes of $\sigma_{x}^{2}$ or of any of the error variances or covariances, we could construct a generalization of the "errors in variables" estimator. As in the previous section, however, we will assume that we do not have any plausible restrictions. In this case the issue is how to optimally use the information contained in the proxies in order to minimize the attenuation bias.

\subsection{Minimizing attenuation bias}

Let

$$
x^{\delta}=X \delta
$$

be any linear combination of the proxy variables where $X=\left[\begin{array}{llll}x_{1} & x_{2} & \ldots & x_{k}\end{array}\right]$. By assumption

$$
X=x \rho^{\prime}+U
$$

where $\rho^{\prime}=\left[\begin{array}{cccc}1 & \rho_{2} & \ldots & \rho_{k}\end{array}\right]$. It follows that

$$
x^{\delta}=x \rho^{\prime} \delta+U \delta
$$

Unless $\rho^{\prime} \delta=1$ this will involve a rescaling, so we will want to multiply our final estimates by $\rho^{\prime} \delta$ to make all results comparable. 
Theorem 1 Let $b^{\delta}=\widehat{\beta}\left(\rho^{\prime} \delta\right)$ be the rescaled OLS estimate in the regression of $y$ on $x^{\delta}$, $b$ be the OLS estimate in the regression of $y$ on $X$, and $\hat{\rho}$ be the GMM estimate of $\rho$. Then

$$
\begin{aligned}
p \lim b^{\delta} & =\beta\left(1-\frac{\delta^{\prime} \Sigma_{U U} \delta}{\sigma_{x}^{2}\left(\delta^{\prime} \rho\right)^{2}+\delta^{\prime} \Sigma_{U U} \delta}\right) \\
\operatorname{plim} \widehat{\rho}^{\prime} b & =\beta\left(1-\frac{\left|\Sigma_{U U}\right|}{\left|\Sigma_{X X}\right|}\right) \\
& =\beta\left(1-\frac{1}{\sigma_{x}^{2} \rho^{\prime} \Sigma_{U U}^{-1} \rho+1}\right)
\end{aligned}
$$

where $\Sigma_{X X}$ is the covariance matrix of $X$. Furthermore for every $\delta \neq 0$ we have

$$
\frac{1}{\sigma_{x}^{2} \rho^{\prime} \Sigma_{U U}^{-1} \rho+1} \leq \frac{\delta^{\prime} \Sigma_{U U} \delta}{\sigma_{x}^{2}\left(\delta^{\prime} \rho\right)^{2}+\delta^{\prime} \Sigma_{U U} \delta}
$$

Equality holds only if

$$
\delta=c \Sigma_{U U}^{-1} \rho
$$

for some $c \neq 0$.

This theorem contains the generalization of the discovery we made in the two proxy case with $\rho_{2}=1$. We note that the appropriate way of aggregating up the coefficients in the multiple regression is given by the "post hoc" estimator

$$
b^{p}=\hat{\rho}^{\prime} b=\sum_{j=1}^{k} \frac{\operatorname{cov}\left(y, x_{j}\right)}{\operatorname{cov}\left(y, x_{1}\right)} b_{j}
$$

where $b_{j}$ is the coefficient on $x_{j}$ in the multiple regression. We use the term post hoc both because the estimation happens after the event (the regression), but also because it can be seen as a rationalization of the data: from the $k$ different regression coefficients on the proxies, the post hoc estimators gives a way to interpret how changes in the underlying unobserved variable $x$ effect the dependent variables. The coefficients on the proxies themselves have the less straightforward interpretation of the effect of a unit change in the proxy holding all other proxies constant.

The theorem proves that no linear combination of the proxies will achieve a greater reduction in attenuation bias than our process of post hoc inference. The theorem therefore covers all of the procedures outlined in the introduction: averaging, standardizing and then adding, and construction of the first principal component. In essence the multiple regression provides the appropriate reweighting of the variables to minimize the error variance of the aggregate set of proxies. 
We note that the formula in equation 10 provides the natural generalization of equation 2, with the "generalized variances" $\left|\Sigma_{U U}\right|$ and $\left|\Sigma_{X X}\right|$ replacing $\sigma_{1}^{2}$ and $\sigma_{x}^{2}$ respectively (see Dhrymes 1974, p.56).

\subsection{Post hoc inference and index construction}

Our procedure obviously depends on the validity of the underlying assumptions. If the proxies belong in the main regression (equation 1a), then clearly the process of aggregating up the coefficients will not correspond to any parameter of interest. Nevertheless the procedure is more robust to departures from the validity of the underlying assumptions than index construction prior to estimating the regression will be.

One attractive feature is that it is possible to provide the reader with the estimates of the $\rho$ s and the reader can then assess how plausible the assumption of the "common factor" is. For example, if the latent variable is "wealth", it would be strange if the number of rooms in one's house did not load strongly on to it. Furthermore, given the $\rho \mathrm{s}$, it is possible to provide different estimates of $\beta$, depending on whether particular proxies are viewed as having independent effects or not.

Strictly speaking our procedure should therefore not be viewed as an estimation but as an interpretation procedure. Equation 11 tells us how to interpret the coefficients of a multiple regression under the null hypothesis that there is a common underlying factor which is generating the separate coefficients.

Another way in which we can interpret the procedure is as a particular way of constructing a composite index from the separate proxies. Indeed, as Theorem 1 shows, there will always be one linear combination of the variables that will provide exactly the same coefficient as the estimator (11). The multiple regression can therefore be viewed as implicitly constructing an index from the separate proxies. Our procedure provides the coefficient on this index. Indeed we can make this implicit index explicit post hoc as well:

$$
x^{p}=\frac{1}{b^{p}} \sum_{j=1}^{k} x_{j} b_{j}
$$

where $b_{j}$ is the $j$-th regression coefficient. By construction this index is on the same scale as $x_{1}$ and will reproduce $b^{p}$ as the coefficient in the regression. ${ }^{1}$

\footnotetext{
${ }^{1}$ We have $\delta=\frac{1}{b^{p}} b$ with $b=\left(X^{\prime} X\right)^{-1} X^{\prime} y$. Consequently $\delta^{\prime} \rho=1\left(\right.$ since $\left.b^{p}=b^{\prime} \rho\right)$ and $\left(\delta^{\prime} X^{\prime} X \delta\right)^{-1} \delta^{\prime} X^{\prime} y=b^{p}$.
} 
We are not proposing this post hoc index as the "magic bullet" for dealing with proxies, but it has the virtue of allowing us to do various checks on the plausibility of the procedure. But obviously this index is not a general purpose one - it is that common factor in the proxies which best explains $y$. In a different regression, a different index would be selected by the procedure. One should therefore be cautious in identifying the index with the underlying latent variable.

If we view the regression as a procedure for implicitly constructing an index, then the individual regression coefficients have the interpretation as weights. From the final condition in Theorem 1 it is clear that this reweighting must work so that the weight is proportional to the correlation with $x$ and (in a sense) inversely proportional to the error variance. The multiple regression procedure must therefore "parcel out" the overall regression coefficient $b^{p}$ proportional to $\rho$ and inversely proportional to the error variance. We can show this somewhat more precisely.

Proposition 2 Let $b_{i}$ be the $i$-th regression coefficient in the multiple regression of $y$ on $X$, i.e.

$$
b_{i}=e_{i}^{\prime}\left(X^{\prime} X\right)^{-1} X^{\prime} y
$$

where $e_{i}$ is the unit vector with one in the $i$-th position. Then

$$
\operatorname{plim}_{n \rightarrow \infty} b_{i}=\beta \frac{\sigma_{x}^{2}\left|\Sigma_{U U}^{\rho(i)}\right|}{\left|\Sigma_{X X}\right|}
$$

where $\Sigma_{U U}^{\rho(i)}$ is the matrix obtained by deleting row $i$ of $\Sigma_{U U}$ and replacing it with the vector $\rho^{\prime}$.

In the special case where $\Sigma_{U U}$ is the diagonal matrix, it follows that

$$
\begin{aligned}
\operatorname{plim} \frac{b_{i}}{b_{j}} & =\frac{\rho_{i} \prod_{k \neq i} \sigma_{k}^{2}}{\rho_{j} \prod_{k \neq j} \sigma_{k}^{2}} \\
& =\frac{\rho_{i} \sigma_{j}^{2}}{\rho_{j} \sigma_{i}^{2}}
\end{aligned}
$$

Several additional points follow from this result. Firstly, if $\beta=0$, then every single proxy coefficient must be zero. This means that the hypothesis that $\beta=0$ is testable as a joint hypothesis on all the proxies. Indeed, it is also testable on the sum of the proxies.

Secondly, it follows from our proof about the bias in $\rho^{\prime} b$ that

$$
\left|\Sigma_{X X}\right|=\sum_{i=1}^{k} \rho_{i} \sigma_{x}^{2}\left|\Sigma_{U U}^{\rho(i)}\right|+\left|\Sigma_{U U}\right|
$$


There are therefore $k+1$ terms in the denominator of equation 13 . If the proxies are all of similar quality, i.e. if the $\rho$ s and error variances are not vastly dissimilar, then the individual coefficients should be of the order of $\frac{\beta}{k}$, i.e. as more proxies are added, the individual coefficients should tend to zero. It is this feature that possibly accounts for researchers apprehension in adding multiple noisy measures of the same variable into a regression. It should be clear, however, that this is not the appropriate metric in which to think about the size of the coefficient. It is not the individual contributions that matter, but the aggregate one.

\subsection{The impact of other covariates}

It is well-known that the attenuation bias in the OLS coefficient on a mismeasured variable is increased when correctly measured variables are also included in the model, provided that these variables are not correlated with the measurement error (Griliches 1986). Furthermore the bias is transmitted to the coefficients of the correctly measured variables, generally with the opposite sign. Both of these results also apply to the bias in the post hoc estimator when multiple proxies for an unobserved variable are included in the regression. The coefficients on the covariates will be biased as well, the magnitude of which depends on the covariances between the covariates, the unobserved variable, and the measurement error components in the proxies $\left(u_{j}\right.$ above).

In this case, however, it is particularly important to be concerned about the correlation between the measurement error component and the covariates. Adding in proxies that absorb the effects of the covariates instead of proxying for the latent variable would be particularly damaging. An important trade-off exists, therefore, in adding additional proxies that may add little information about the underlying unobserved variable, but affect the accuracy with which we measure the coefficients on correctly measured variables in the model. The post hoc interpretation procedure should therefore not be taken as licence to throw any and all variables into the regression. Ideally, the proxies should be correlated with $x$ and their measurement error components should be orthogonal to the other explanatory variables in the regression. 


\subsection{Biased instrumental variables estimation}

The results above indicate that our post hoc approach is superior to the ad hoc index construction approaches seen in the literature. Nevertheless it is not clear that it is the best approach possible. For instance, if the error components in the proxies are mutually independent, then instrumental variables will deliver an unbiased estimate of the structural parameter. One might speculate, therefore, that biased IV estimation in cases where the errors in the proxies are only weakly correlated might still do better than the post hoc approach. Indeed we can investigate under which circumstances this is likely to be the case.

In the two variable case given in equations $1 \mathrm{a}-1 \mathrm{c}$, if we use $x_{2}$ as an instrument for $x_{1}$, then asymptotically

$$
\begin{aligned}
b_{i v} & =\frac{\operatorname{cov}\left(x_{2}, y\right)}{\operatorname{cov}\left(x_{2}, x_{1}\right)} \\
& =\beta \frac{\sigma_{x}^{2}}{\sigma_{x}^{2}+\frac{\sigma_{12}}{\rho_{2}}}
\end{aligned}
$$

It is obvious that the smaller the covariance between $u_{1}$ and $u_{2}$ is, the smaller the asymptotic bias. Unlike in the least squares case, however, the direction of the bias depends on the sign of $\sigma_{12}$. There is no longer a guarantee that the estimate is a lower bound on the true value.

If we let $\rho_{2}=1$ we can compare the absolute value of the IV bias to the bias in the estimate of $b^{*}$ (equation 3). Instrumental variables will yield a smaller absolute bias if, and only if

$$
\left|\sigma_{12}\right| \leq \frac{\sigma_{2}^{2} \sigma_{1}^{2}-\sigma_{12}^{2}}{\sigma_{1}^{2}-2 \sigma_{12}+\sigma_{2}^{2}}
$$

If $\sigma_{12}>0$ we get the condition

$$
0 \leq \sigma_{2}^{2} \sigma_{1}^{2}-\left(\sigma_{1}^{2}+\sigma_{2}^{2}\right) \sigma_{12}+\sigma_{12}^{2}
$$

This quadratic in $\sigma_{12}$ is guaranteed to have real roots. The condition will be satisfied if, and only if

$$
0 \leq \sigma_{12} \leq \min \left(\sigma_{1}^{2}, \sigma_{2}^{2}\right) \text { or } \sigma_{12} \geq \max \left(\sigma_{1}^{2}, \sigma_{2}^{2}\right)
$$


The latter condition is irrelevant, since the positive definiteness of $\Sigma_{U U}$ implies that $\sigma_{12} \leq \frac{\sigma_{1}^{2}+\sigma_{2}^{2}}{2}$. The condition therefore simplifies to

$$
\sigma_{12} \leq \min \left(\sigma_{1}^{2}, \sigma_{2}^{2}\right)
$$

It follows that if the error variances are positively correlated, biased IV will be superior provided the error variances are of similar magnitudes

If $\sigma_{12}<0$ we get the condition

$$
3 \sigma_{12}^{2}-\left(\sigma_{1}^{2}+\sigma_{2}^{2}\right) \sigma_{12}-\sigma_{2}^{2} \sigma_{1}^{2} \leq 0
$$

which gives

$$
\frac{\sigma_{1}^{2}+\sigma_{2}^{2}}{6}-\frac{\sqrt{\sigma_{1}^{4}+14 \sigma_{1}^{2} \sigma_{2}^{2}+\sigma_{2}^{4}}}{6} \leq \sigma_{12} \leq 0
$$

In the particular case where the error variances are equal, this condition is equivalent to $\sigma_{12} \geq-\frac{1}{3}\left(\sigma_{u}^{2}\right)$ where $\sigma_{u}^{2}$ is the common error variance.

Combining the two cases we find that biased IV is superior if, and only if,

$$
\frac{\sigma_{1}^{2}+\sigma_{2}^{2}}{6}-\frac{\sqrt{\sigma_{1}^{4}+14 \sigma_{1}^{2} \sigma_{2}^{2}+\sigma_{2}^{4}}}{6} \leq \sigma_{12} \leq \min \left(\sigma_{1}^{2}, \sigma_{2}^{2}\right)
$$

It is evident that in many situations biased IV will improve on ordinary least squares. If we have more than two proxies, there is yet further scope for improvement. In this case, however, the problem is to find the linear combination of proxies where the error is least correlated with the error in $x_{1}$. The conventional two-stage least squares estimate (using all the proxies as instruments for $x_{1}$ ) will definitely fare badly in this regard, since they will seek to explain not only the part of $x_{1}$ which is correlated with $x$, but also the error term $u_{1}$.

We can put the problem more formally as follows: let $\gamma=\left[0, \gamma_{2}, \ldots, \gamma_{k}\right]^{\prime}$ be a column vector of real numbers with $\gamma_{1}=0$, so that $X \gamma$ is an arbitrary linear combination of $x_{2} \ldots x_{k}$. Using $X \gamma$ as an instrument for $x_{1}$ yields asymptotically

$$
\begin{aligned}
b_{i v}^{\gamma} & =\beta \frac{\sigma_{x}^{2} \rho^{\prime} \gamma}{\sigma_{x}^{2} \rho^{\prime} \gamma+\sum_{i=2}^{k} \gamma_{i} \sigma_{1 i}} \\
& =\beta \frac{\sigma_{x}^{2}}{\sigma_{x}^{2}+\frac{1}{\rho^{\prime} \gamma} \sum_{i=2}^{k} \gamma_{i} \sigma_{1 i}}
\end{aligned}
$$

The "best" instrument is that linear combination which yields the smallest absolute value of $\frac{1}{\rho^{\prime} \gamma} \sum_{i=2}^{k} \gamma_{i} \sigma_{1 i}$. We can rewrite this expression as $\sum_{i=2}^{k} \omega_{i} \frac{\sigma_{1 i}}{\rho_{i}}$, where $\omega_{i}=\frac{\rho_{i} \gamma_{i}}{\sum \rho_{i} \gamma_{i}}$, 
so that we are looking for a "weighted average" of the terms $\frac{\sigma_{1 i}}{\rho_{i}}$. Unfortunately neither the weights nor the terms need to be positive, so there is insufficient information for the empirical researcher to gauge how to construct such an instrument. Even if such an instrument could be constructed, it would yield a smaller absolute bias only if

$$
\left|\frac{1}{\rho^{\prime} \gamma} \sum_{i=2}^{k} \gamma_{i} \sigma_{1 i}\right| \leq \frac{1}{\rho^{\prime} \Sigma_{U U}^{-1} \rho}
$$

With the information available to an empirical researcher it is impossible to construct the appropriate instrument, ascertain whether the condition above is satisfied, or what the direction of the bias is. Consequently reporting the post hoc least squares estimates jointly with any IV estimates is likely to be a preferred strategy.

\section{Monte Carlo simulations}

The results above are all asymptotic. In finite samples we are faced by a number of problems. Firstly, we need to estimate the $\rho$ s, and this will increase the noise in our procedure. Secondly, there are trade-offs between degrees of freedom lost from including too many proxies and the increased precision gained by putting them all in.

To investigate these issues we run a Monte Carlo simulation. While the relative performance of different estimators will depend on the parameters we set for the simulations, by knowing the true data generating process we are able to assess the overall bias in all estimates. In section 5 we take a different approach and compare the estimators using actual datasets.

For each of 100 runs, we draw 100 independent observations on $x$ and $\varepsilon$ from $\mathrm{N}(0,2)$, and then create $y$ equal to $10+100 * x+\varepsilon$. We generate 20 proxy variables for $x$ determined by $x_{j}=\rho_{j} x+u_{j}$ with the properties that each proxy has a different correlation $\left(\rho_{j}\right)$ with the unobserved factor $x$, has a different error variance $\left(u_{j}\right)$, and the error components are correlated with each other. Specifically, $\rho_{1} \equiv 1$, and for $j=2$ to $20, \rho_{j}$ is randomly drawn from $\mathrm{U}(0,2) . \operatorname{Var}\left(u_{j}\right)=1.1^{j-1}$ and $\mathrm{E}\left(u_{j} u_{k}\right)=0.5^{k-j} \sigma_{j} \sigma_{k}$ for $j \neq k . \rho$ and the covariance structure of the $u_{j}$ s are fixed for the 100 runs. We randomly reorder the proxies before each run to avoid systematically changing the quality of the proxies as we successively add them to the model. To ensure that our inferences about different estimators are not driven by the particular $\rho$ vector we drew, the 100 simulations are

repeated ten times with ten different draws of $\rho$, and the results averaged together. 
Table 1 displays the mean and standard deviation of five estimators. Each row indicates the number of proxies used in the model. The mean square errors of the final model, which uses all 20 proxies, is given at the bottom of the table. The first column shows results when $y$ is regressed on all of the proxies and the coefficients are averaged together weighted by the true value of $\rho$. In the second column the coefficients are weighted by an estimate of $\rho, \hat{\rho}_{j}=\frac{\operatorname{cov}\left(y, x_{j}\right)}{\operatorname{cov}\left(y, x_{1}\right)}$. Since $\rho_{1} \equiv 1$ the estimates with only one proxy are identical, 66.77, which is biased downwards by 33 percent. As more proxies are added, the estimates based on the true $\rho$ and the estimated $\rho$ remain very close to each other; with 10 proxies the bias is about 10 percent in each and with 20 proxies it 5.7 and 4 percent. Since $\rho$ can be consistently estimated, the only cost in having to do so is the additional imprecision in the estimate. With 20 proxies the standard error rises from 2.8 to 7.2 as a result of having to estimate $\rho$. Note that the bias is slightly smaller in the second column than in the first.

The third through fifth columns implement alternative estimators that have appeared in the literature. In these we regress $y$ on the unweighted average of the proxies, on the average of proxies after they have been standardized to have a mean of zero and unit variance, and on the first principal component of the proxies. We rescale the results for these estimators as appropriate to be comparable to those in the first two columns. All three of these estimator perform considerably poorer than the post hoc estimators in the first two columns. Although additional proxies improve each estimator, with 20 variables the bias in each is $18.5,20.6$, and 14.5 percent respectively. Since these estimates do not require the additional estimation of the weights placed on each proxy, there is a small gain in the precision of the estimates, but as the last row shows, the mean squared error is still between 3.5 and seven times as large as the that in second column. We conclude from these simulations that in situations where one cannot be confident that the proxies are of similar quality (i.e. similar $\rho$ s and error variances), the reduction in bias from aggregating the coefficients on the proxies can be considerable, compared to entering a single, essentially arbitrary combination of the proxies in the regression. 


\section{Applications to existing research}

We illustrate the procedure with two empirical examples. Though we do not know the true data generating process, and thus cannot compare the alternative estimators against the true parameter values, the use of actual data allows us to assess whether the alternative estimators themselves produce qualitatively different results. In the first example we are interested in estimating the effect of a family's permanent income on their children's performance on a reading comprehension test. Permanent income is not observed, and we instead use panel data with up to nine years of annual income as alternative noisy measures of permanent income. In the second example we use data on assets and housing conditions from the Demographic and Health Survey of India as proxies for household wealth in a model linking wealth and school attendance. Filmer and Pritchett (2001) use the first principal component of the asset variables as their measure of wealth.

A standard model of $(\log )$ permanent income specifies observed income in year $t$ as being composed of unobserved permanent and transitory or luck components: $y_{t}=$ $y_{p}+u_{t}$. If parents' investment in their children is a function of their permanent income, then the correlation between observed income understate the true correlation in the permanent incomes of parents and their children. To circumvent this problem, a general practice in the literature has been to average annual income over several years. See, for example, Blau (1999), Case et al (2001), Mayer (1997), Solon (1992), and Zimmerman (1992).

Using data from the National Longitudinal Survey of Youth (NLSY), we examine the relationship between family income and children's percentile score on the Peabody Individual Achievement Test in reading comprehension. The NLSY began in 1979 with a sample of 12,686 individuals aged 14 to 21 . Interviews were conducted annually between 1979 and 1994, and biennially since then. In 1986 a separate biennial survey of the children of the women from the 1979 cohort began (called the NLSY-Children). Our sample contains 5232 children-year observations of those aged six to fourteen who have nonmissing data on current and nine lags of family income. The model also includes controls for the log of family size, the child's sex, age, and race, the mother's age and education, whether the mother's spouse is present, and if so, his age and education, year 
effects, and the mother's age-adjusted AFQT score (a test of reading and math skills that was administered to the mother in 1980). We drop children who have missing data for any of these controls.

Figure 1 plots the results from different models of children's test scores. Following the common practice in the literature, our first measure of permanent income is to average log income over several time periods. The line in the figure labeled "Using average income" indicates the coefficients on this term when it contains current and from zero to nine lags of income. The coefficient rises from 0.339 when only current income is used to 1.183 when current income and four lagged values are average together. The coefficient continues to rise by small amounts as additional lags beyond the fourth are introduced.

Next we include current and lagged values of family income in the regression separately, and average the coefficients first unweighted, and second weighted by the GMM estimate of $\rho$. The estimates of $\rho$ are also given in the table and they show a small rise from the first to the fourth income lag, going from 1.0 to 1.15, after which there is a slight decrease back to one. The unweighted average of the regression coefficients assumes that the correlation between the test score and current and each lag of income are equal, a restriction the data in fact reject. These estimates nearly match the estimated effect when the income data are averaged together prior to the regression. The only difference between these estimates is caused by differences in the variance of the transitory income component across different lags, which does not seem to be an important feature of this data. When the income lags are optimally weighted, the total effect of permanent income grows by 17 to 19 percent compared to unweighted average effect. The estimated effect of permanent income when current and all nine lags of annual income are used is 1.613 , implying that a 50 percent rise in income leads to about a 0.8 percentile points higher reading score.

Our second empirical application reexamines Filmer and Pritchett's attempt to estimate the effect of household wealth on Indian children's propensity to be enrolled in school. The catch is that the Demographic and Health Survey of India does not contain any income or wealth data, but it does contain many questions on asset holdings and dwelling quality. Filmer and Pritchett propose to use the first principal component of 
these asset variables as their measure of wealth.

We use data on 109,973 children aged 4 to 16 with nonmissing data for all variables. The dependent variable in the regression is an indicator that the child is enrolled in school. The asset variables are the number of rooms in the house, indicators for whether the household has a refrigerator, clock or watch, sewing machine, VCR, radio, television, fan, bicycle, car, motorcycle, electric lighting, a flush toilet or latrine, livestock; whether the kitchen is in a separate room in the house, whether the primary cooking fuel is wood, cow dung, or coal, and whether the drinking and nondrinking water comes from a pump or an open source (as opposed to being piped into the home).

The first column of Table 2 displays $\rho$, the ratios of the bivariate correlations between each asset and the school enrollment indicator, to the correlation between the number of rooms in the house and the indicator. Thus the units of our unobserved "wealth" index is the number of rooms in the dwelling. Assets that are more likely among poorer households, such as those who obtain water from pumps or an open source, use wood, cow dung, or coal as cooking fuel, or own livestock, have negative correlations with children's school enrollment. The number of rooms in the house has the largest correlation; The assets one would associate with the relatively best-off in the data having a car, VCR, or refrigerator - are are only owned by a small proportion of the household, and thus do very poorly in accounting for enrollment rates among the whole population.

The next six columns show results when all or some of the asset variables are entered separately into the regression. The model also controls for the child's sex and age, the head of the household's sex, age, and education, and the log family size. Nearly all of the asset variables are statistically significant, although some, such as refrigerator, car, and VCR ownership, and using wood, dung, or coal as cooking fuel, have a different sign than their raw correlation with school enrollment. One might be tempted to drop these variables from the model, thinking they are capturing something other than the effect of wealth on school enrollment. As illustrated in equation 4, a proxy that is highly correlated with another, better measured proxy may well have a different sign than the true effect to be measured.

When all 18 asset variables are used, the estimated effect of the assets is $0.170 .^{2}$ To

\footnotetext{
${ }^{2}$ Although there are 21 separate variables, we label the two indicating toilet types, and the sources of drinking
} 
see how sensitive the estimate is to using less proxies, in the next five columns we break the 18 proxies into two groups of nine, and then three groups of six. When nine are used, the effects are 0.136 and 0.132 ; when six are used the effects are $0.129,0.105$, and 0.116. The attenuation bias in the estimates clearly increases as less proxies are used. The estimates with the same number of proxies are remarkably close to each other, suggesting the assumption of a single unobserved factor is plausible.

The last column of Table 2 displays the scoring vector used to weight the asset variables for the first principal component. These have been divided by the weight for the number of rooms in the house, so their magnitudes are comparable to the $\rho$ 's estimated below. The coefficient on the principal component asset index is 0.050 . We

rescale this coefficient by multiplying it by $\frac{\rho^{\prime} \delta}{\sigma_{p c}}$ to make it comparable to the estimates where the assets are entered separately. In this formula $\rho$ is the bivariate correlations between each asset and school enrollment, $\delta$ are the scoring factors, and $\sigma_{p c}$ is a vector of the standard deviations of the asset variables. The adjusted coefficient on the asset index is 0.098 , over 40 percent smaller than the effect estimated when all the proxies are entered separately and their coefficients recombined. Indeed, the estimate of the first principal component from all 18 asset variables has more attenuation bias than any of the post hoc estimators that use only six of the assets.

\section{Conclusion}

We have proposed a new estimator for the case where the researcher has multiple proxies for a single, unobservable independent variable. Numerous previous studies have dealt with the problem either by using the proxies one at a time, or by averaging or otherwise aggregating the proxies together and using that single measure as an independent variable. We show that attenuation bias is maximally reduced when the proxies are entered simultaneously in a multiple regression, and the coefficients on them optimally combined after the fact to yield an estimate of the effect of the unobserved variable. To optimally weight the proxies prior to the regression requires knowing the variances and covariances between the error components in the proxies, information that is simply unavailable to the researcher. The improved performance of the post

and nondrinking water as each being one, rather than two, proxies. 
hoc estimator is due to the fact that the regression coefficients on the proxies precisely reflect this unknown information, leaving the researcher only to combine coefficients. This method is also more transparent than ad hoc index construction because a reader who believes some proxies have independent effects on the dependent variable has the information available to create alternative estimates based on a subset of the proxies.

We have put off discussion of the asymptotic or finite-sample distribution of the estimators compared in this paper. The need to estimate $\rho$, the covariances between the proxies and the unobserved factor, introduces additional noise into the estimates that is not present in an ad hoc index variable. The Monte Carlo simulation presented in Section 4 suggest that this source of variance may not be particularly large, and is outweighed by the large reduction in bias in the estimates themselves. More generally, the analytic distribution of the estimators is quite difficult to compute and researchers are probably better off using bootstrap methods to calculate the standard error of their estimates. 


\section{References}

Aigner, Dennis J., Cheng Hsiao, Arie Kapteyn, and Tom Wansbeek, "Latent Variable Models in Econometrics," in Zvi Griliches and Michael D. Intriligator, eds., Handbook of Econometrics, Vol. II, Elsevier, 1984, pp. 1321-1393.

Bertrand, Marianne and Sendhil Mullainathan, "Do people mean what they say? Implications for subjective survey data," American Economic Review Papers and Proceedings, 2001, 91 (2), 67-72.

Blau, David M., "The Effect of Income on Child Development," The Review of Economics and Statistics, 1999, 81 (2), 261-276.

Case, Anne, Darren Lubotsky, and Christina Paxson, "Economic Status and Health in Childhood: The Origins of the Gradient," Working paper W8344, National Bureau of Economic Research June 2001.

Dhrymes, Phoebus J., Econometrics: Statistical Foundations and Applications, New York: Springer, 1974.

Filmer, Deon and Lant H. Pritchett, "Estimating Wealth Effects Without Expenditure Data-Or Tears: An Application to Educational Enrollment in States of India," Demography, February 2001, 38 (1), 115-132.

Fuller, Wayne A., Measurement Error Models, New York: Wiley, 1987.

Glaeser, Edward L., David I. Laibson, José A. Scheinkman, and Christine L. Soutter, "Measuring Trust," Quarterly Journal of Economics, 2000, 115, 811-846.

Goldberger, Arthur S., "Structural Equation Methods in the Social Sciences," Econometrica, 1972, 40 (6), 979-1001.

Griliches, Zvi, "Economic Data Issues," in Zvi Griliches and Michael D. Intriligator, eds., Handbook of Econometrics, Vol. III, Elsevier, 1986, pp. 1466-1514.

Jöreskog, Karl G. and Arthur S. Goldberger, "Estimation of a Model with Multiple Indicators and Multiple Causes of a Single Latent Variable," Journal of the American Statistical Association, 1975, 70 (351), 631-639.

Leamer, Edward E., "Model Choice and Specification Analysis," in Zvi Griliches and Michael D. Intriligator, eds., Handbook of Econometrics, Vol. I, North-Holland, 1983, pp. 285-330.

Mauro, Paolo, "Corruption and Growth," Quarterly Journal of Economics, 1995, 110 (3), 681-712.

Mayer, Susan E., What Money Can't Buy: Family Income and Children's Life Chances, Cambridge, MA: Harvard University Press, 1997.

Solon, Gary, "Intergenerational Income Mobility in the United States," American Economic Review, June 1992, 82 (3), 393-408.

Sydsaeter, Knut, Arne Strom, and Peter Berck, Economists' Mathematical Manual, 3 ed., Berlin: Springer, 1999.

Zimmerman, David J., "Regression Toward Mediocrity in Economic Stature," American Economic Review, June 1992, 82 (3), 409-429. 


\section{A Proofs}

Lemma A.1 $\left|A B+I_{m}\right|=\left|B A+I_{n}\right|$ where $A$ is $m \times n$ and $B$ is $n \times m$. (Sydsaeter, Strom and Berck 1999, p.133, result 20.14)

\section{Proof of Theorem 1.}

$$
\operatorname{plim} \widehat{\beta}=\left(\operatorname{plim} \frac{1}{n}\left(x^{\delta \prime} x^{\delta}\right)\right)^{-1}\left(\operatorname{plim} \frac{1}{n} x^{\delta \prime} y\right)
$$

Now

$$
\operatorname{plim} \frac{1}{n}\left(x^{\delta \prime} x^{\delta}\right)=\sigma_{x}^{2}\left(\rho^{\prime} \delta\right)^{2}+\delta^{\prime} \Sigma_{U U} \delta
$$

and

$$
\operatorname{plim} \frac{1}{n} x^{\delta \prime} y=\beta \delta^{\prime} \rho \sigma_{x}^{2}
$$

Consequently

$$
\begin{aligned}
\operatorname{plim} \widehat{\beta} & =\beta \frac{\sigma_{x}^{2} \delta^{\prime} \rho}{\sigma_{x}^{2}\left(\delta^{\prime} \rho\right)^{2}+\delta^{\prime} \Sigma_{U U} \delta} \\
& =\frac{\beta}{\delta^{\prime} \rho}\left(1-\frac{\delta^{\prime} \Sigma_{U U} \delta}{\sigma_{x}^{2}\left(\delta^{\prime} \rho\right)^{2}+\delta^{\prime} \Sigma_{U U} \delta}\right)
\end{aligned}
$$

i.e.

$$
b^{\delta}=\beta\left(1-\frac{\delta^{\prime} \Sigma_{U U} \delta}{\sigma_{x}^{2}\left(\delta^{\prime} \rho\right)^{2}+\delta^{\prime} \Sigma_{U U} \delta}\right)
$$

By contrast

$$
\begin{aligned}
\operatorname{plim} \hat{\rho}^{\prime} b & =(\operatorname{plim} \hat{\rho})^{\prime}\left(\operatorname{plim} \frac{1}{n} X^{\prime} X\right)^{-1}\left(\operatorname{plim} \frac{1}{n} X^{\prime} y\right) \\
& =\rho^{\prime}\left(\Sigma_{X X}\right)^{-1} \rho \beta \sigma_{x}^{2}
\end{aligned}
$$

Now

$$
\begin{array}{rlr}
\rho^{\prime}\left(\Sigma_{X X}\right)^{-1} \rho \sigma_{x}^{2}-1 & =\left|\sigma_{x}^{2} \rho^{\prime}\left(\Sigma_{X X}\right)^{-1} \rho-1\right| & \quad \text { (determinant of a } 1 \times 1 \text { matrix }) \\
& =\left|\left(\Sigma_{X X}\right)^{-1} \sigma_{x}^{2} \rho \rho^{\prime}-I_{k}\right| \quad \text { (By lemma A.1) } \\
& =\left|\Sigma_{X X}^{-1}\right|\left|\sigma_{x}^{2} \rho \rho^{\prime}-\Sigma_{X X}\right| \\
& =\left|\Sigma_{X X}^{-1}\right|\left|\sigma_{x}^{2} \rho \rho^{\prime}-\sigma_{x}^{2} \rho \rho^{\prime}-\Sigma_{U U}\right| \\
& =\left|\Sigma_{X X}^{-1}\right|\left|-\Sigma_{U U}\right|
\end{array}
$$


i.e.

$$
\rho^{\prime}\left(\Sigma_{X X}\right)^{-1} \rho \sigma_{x}^{2}=1-\frac{\left|\Sigma_{U U}\right|}{\left|\Sigma_{X X}\right|}
$$

Hence

$$
\operatorname{plim} \hat{\rho}^{\prime} b=\beta\left(1-\frac{\left|\Sigma_{U U}\right|}{\left|\Sigma_{X X}\right|}\right)
$$

Now

$$
\begin{aligned}
\left|\Sigma_{X X}\right| & =\left|\sigma_{x}^{2} \rho \rho^{\prime}+\Sigma_{U U}\right| \\
& =\left|\Sigma_{U U}\right|\left|\sigma_{x}^{2} \Sigma_{U U}^{-1} \rho \rho^{\prime}+I_{k}\right| \\
& =\left|\Sigma_{U U}\right|\left|\sigma_{x}^{2} \rho^{\prime} \Sigma_{U U}^{-1} \rho+1\right| \quad \quad \text { (by lemma A.1) } \\
& =\left|\Sigma_{U U}\right|\left(\sigma_{x}^{2} \rho^{\prime} \Sigma_{U U}^{-1} \rho+1\right)
\end{aligned}
$$

hence

$$
\frac{\left|\Sigma_{U U}\right|}{\left|\Sigma_{X X}\right|}=\frac{1}{\sigma_{x}^{2} \rho^{\prime} \Sigma_{U U}^{-1} \rho+1}
$$

We want to compare $\frac{1}{\sigma_{x}^{2} \rho^{\prime} \Sigma_{U U}^{-1} \rho+1}$ and $\frac{\delta^{\prime} \Sigma_{U U} \delta}{\sigma_{x}^{2}\left(\delta^{\prime} \rho\right)^{2}+\delta^{\prime} \Sigma_{U U} \delta}$, but

$$
\frac{\delta^{\prime} \Sigma_{U U} \delta}{\sigma_{x}^{2}\left(\delta^{\prime} \rho\right)^{2}+\delta^{\prime} \Sigma_{U U} \delta}=\frac{1}{\sigma_{x}^{2} \frac{\left(\delta^{\prime} \rho\right)^{2}}{\delta^{\prime} \Sigma_{U U} \delta}+1}
$$

so we need to show that

$$
\rho^{\prime} \Sigma_{U U}^{-1} \rho \geq \frac{\left(\delta^{\prime} \rho\right)^{2}}{\delta^{\prime} \Sigma_{U U} \delta}
$$

for any non-zero choices of $\rho$ and $\delta$.

Since $\Sigma_{U U}$ is a non-singular covariance matrix, by the spectral theorem for symmetric matrices it can be decomposed as

$$
\Sigma_{U U}=P D P^{\prime}
$$

where $P$ is an orthogonal matrix of eigenvectors

$$
P=\left[\begin{array}{llll}
p_{1} & p_{2} & \ldots & p_{k}
\end{array}\right]
$$

and $D=\operatorname{diag}\left(\lambda_{1}, \ldots, \lambda_{k}\right)$ is the matrix of eigenvalues, with $\lambda_{i}>0, \forall i$. This is equivalent to writing

$$
\Sigma_{U U}=\lambda_{1} p_{1} p_{1}^{\prime}+\ldots+\lambda_{k} p_{k} p_{k}^{\prime}
$$


and it follows that

$$
\Sigma_{U U}^{-1}=\frac{1}{\lambda_{1}} p_{1} p_{1}^{\prime}+\ldots+\frac{1}{\lambda_{k}} p_{k} p_{k}^{\prime}
$$

Hence

$$
\begin{aligned}
& \rho^{\prime} \Sigma_{U U}^{-1} \rho=\sum_{i} \frac{1}{\lambda_{i}}\left(p_{i}^{\prime} \rho\right)^{2} \\
& \delta^{\prime} \Sigma_{U U} \delta=\sum_{i} \lambda_{i}\left(p_{i}^{\prime} \delta\right)^{2}
\end{aligned}
$$

Now let $p_{i}^{\prime} \rho=w_{i}$ and $p_{i}^{\prime} \delta=v_{i}$. Correspondingly define the vectors $w$ and $v$ as

$$
w=P^{\prime} \rho, v=P^{\prime} \delta
$$

Note that

$$
\delta^{\prime} \rho=v^{\prime} w
$$

(since $P$ is orthogonal), i.e.

$$
\begin{aligned}
\left(\delta^{\prime} \rho\right)^{2} & =\left(\sum_{i} v_{i} w_{i}\right)^{2} \\
& \leq\left(\sum_{i} \sqrt{\lambda_{i}}\left|v_{i}\right| \frac{1}{\sqrt{\lambda_{i}}}\left|w_{i}\right|\right)^{2} \\
& \leq\left(\sum_{i} \lambda_{i} v_{i}^{2}\right)\left(\sum_{i} \frac{1}{\lambda_{i}} w_{i}^{2}\right) \quad \text { (Cauchy-Schwarz inequality) } \\
& =\left(\delta^{\prime} \Sigma_{U U} \delta\right)\left(\rho^{\prime} \Sigma_{U U}^{-1} \rho\right)
\end{aligned}
$$

Equality holds only if

$$
\sqrt{\lambda_{i}} v_{i}=\frac{c}{\sqrt{\lambda_{i}}} w_{i}
$$

for some real number $c$, i.e.

$$
\begin{aligned}
\lambda_{i} v_{i} & =c w_{i} \\
D P^{\prime} \delta & =c P^{\prime} \rho \\
\delta & =c P D^{-1} P^{\prime} \rho \\
& =c \Sigma_{U U}^{-1} \rho
\end{aligned}
$$




\section{Proof of proposition 2.}

$$
\begin{aligned}
\operatorname{plim} b_{i} & =e_{i}^{\prime}\left(\operatorname{plim} \frac{1}{n}\left(X^{\prime} X\right)\right)^{-1}\left(\operatorname{plim} \frac{1}{n} X^{\prime} y\right) \\
& =e_{i}^{\prime} \Sigma_{X X}^{-1} \beta \rho \sigma_{x}^{2}
\end{aligned}
$$

Now

$$
\begin{aligned}
e_{i}^{\prime}\left(\Sigma_{X X}\right)^{-1} \rho \sigma_{x}^{2}-1 & =\left|\sigma_{x}^{2} e_{i}^{\prime}\left(\Sigma_{X X}\right)^{-1} \rho-1\right| \quad \text { (determinant of a } 1 \times 1 \text { matrix) } \\
& =\left|\left(\Sigma_{X X}\right)^{-1} \sigma_{x}^{2} \rho e_{i}^{\prime}-I_{k}\right| \quad \text { By lemma A.1) } \\
& =\left|\Sigma_{X X}^{-1}\right|\left|\sigma_{x}^{2} \rho e_{i}^{\prime}-\Sigma_{X X}\right| \quad \\
& =\left|\Sigma_{X X}^{-1}\right|\left|\sigma_{x}^{2} \rho e_{i}^{\prime}-\sigma_{x}^{2} \rho \rho^{\prime}-\Sigma_{U U}\right| \\
& =\left|\Sigma_{X X}^{-1}\right|\left|\sigma_{x}^{2} \rho\left(e_{i}^{\prime}-\rho^{\prime}\right)-\Sigma_{U U}\right| \\
& =\left|\Sigma_{X X}^{-1}\right|\left|\Sigma_{U U}\right|\left|\sigma_{x}^{2} \Sigma_{U U}^{-1} \rho\left(e_{i}^{\prime}-\rho^{\prime}\right)-I_{k}\right| \\
& =\left|\Sigma_{X X}^{-1}\right|\left|\Sigma_{U U}\right|\left|\sigma_{x}^{2}\left(e_{i}^{\prime}-\rho^{\prime}\right) \Sigma_{U U}^{-1} \rho-1\right| \quad \text { (By lemma A.1) } \\
& =\frac{\left|\Sigma_{U U}\right|\left(\sigma_{x}^{2}\left(e_{i}^{\prime}-\rho^{\prime}\right) \Sigma_{U U}^{-1} \rho-1\right)}{\left|\Sigma_{X X}\right|}
\end{aligned}
$$

Hence

$$
e_{i}^{\prime}\left(\Sigma_{X X}\right)^{-1} \rho \sigma_{x}^{2}=\frac{\left|\Sigma_{X X}\right|+\left|\Sigma_{U U}\right|\left(\sigma_{x}^{2}\left(e_{i}^{\prime}-\rho^{\prime}\right) \Sigma_{U U}^{-1} \rho-1\right)}{\left|\Sigma_{X X}\right|}
$$

Substituting the expression for $\left|\Sigma_{X X}\right|$ in equation 17 into the numerator and simplifying, we get

$$
e_{i}^{\prime}\left(\Sigma_{X X}\right)^{-1} \rho \sigma_{x}^{2}=\frac{\left|\Sigma_{U U}\right|\left(\sigma_{x}^{2} e_{i}^{\prime} \Sigma_{U U}^{-1} \rho\right)}{\left|\Sigma_{X X}\right|}
$$

Now

$$
\Sigma_{U U}^{-1}=\frac{1}{\left|\Sigma_{U U}\right|}\left[\begin{array}{cccc}
S_{11} & S_{12} & \cdots & S_{1 k} \\
S_{21} & S_{22} & \cdots & S_{2 k} \\
\vdots & \vdots & \ddots & \vdots \\
S_{k 1} & S_{k 2} & \cdots & S_{k k}
\end{array}\right]
$$

where $S_{i j}$ is the $i j$-th cofactor of $\Sigma_{U U}$. Consequently

$$
e_{i}^{\prime} \Sigma_{U U}^{-1}=\frac{1}{\left|\Sigma_{U U}\right|}\left[\begin{array}{llll}
S_{i 1} & S_{i 2} & \ldots & S_{i k}
\end{array}\right]
$$

i.e.

$$
e_{i}^{\prime} \Sigma_{U U}^{-1} \rho=\frac{1}{\left|\Sigma_{U U}\right|} \sum_{j} S_{i j} \rho_{j}
$$


The term $\sum_{j} S_{i j} \rho_{j}$, however, is identical to the value of the determinant if row $i$ of matrix $\Sigma_{U U}$ were replaced by $\rho^{\prime}$, i.e.

$$
\sum_{j} S_{i j} \rho_{j}=\left|\Sigma_{U U}^{\rho(i)}\right|
$$

Consequently

$$
e_{i}^{\prime}\left(\Sigma_{X X}\right)^{-1} \rho \beta \sigma_{x}^{2}=\frac{\beta \sigma_{x}^{2}\left|\Sigma_{U U}^{\rho(i)}\right|}{\left|\Sigma_{X X}\right|}
$$




\section{Table 1: Monte Carlo Simulation Results}

\begin{tabular}{cccccc} 
& \multicolumn{3}{c}{ Post hoc estimator } & \multicolumn{3}{c}{$\begin{array}{c}\text { Average of } \\
\text { Number of } \\
\text { proxies }\end{array}$} & $\begin{array}{c}\text { true } \\
\text { p's }\end{array}$ & $\begin{array}{c}\text { estimated } \\
\text { p's }\end{array}$ & $\begin{array}{c}\text { Average of } \\
\text { proxies }\end{array}$ & $\begin{array}{c}\text { standardized } \\
\text { proxies }\end{array}$ & $\begin{array}{c}\text { First principal } \\
\text { component }\end{array}$ \\
\hline 1 & 66.77 & 66.77 & 66.77 & 66.77 & 66.77 \\
& $(4.69)$ & $(4.69)$ & $(4.69)$ & $(4.69)$ & $(4.69)$ \\
2 & 73.14 & 73.52 & 62.62 & 63.71 & 63.45 \\
& $(7.26)$ & $(7.88)$ & $(16.00)$ & $(16.19)$ & $(16.65)$ \\
3 & 77.67 & 78.37 & 66.26 & 66.12 & 69.72 \\
& $(7.39)$ & $(8.40)$ & $(14.96)$ & $(15.01)$ & $(15.10)$ \\
4 & 80.95 & 81.85 & 69.57 & 68.74 & 73.48 \\
& $(6.91)$ & $(8.27)$ & $(13.70)$ & $(13.73)$ & $(12.98)$ \\
5 & 83.37 & 84.40 & 71.97 & 70.58 & 75.94 \\
& $(6.18)$ & $(8.06)$ & $(12.12)$ & $(12.36)$ & $(11.14)$ \\
& & & & & \\
10 & 89.58 & 91.10 & 77.56 & 75.57 & 81.78 \\
& $(4.21)$ & $(7.29)$ & $(8.37)$ & $(8.68)$ & $(7.09)$ \\
& & & & & \\
15 & 92.53 & 94.18 & 80.16 & 78.00 & 84.23 \\
& $(3.26)$ & $(7.21)$ & $(6.71)$ & $(7.13)$ & $(5.50)$ \\
& & & & & \\
20 & 94.26 & 96.00 & 81.46 & 79.36 & 85.47 \\
& $(2.76)$ & $(7.18)$ & $(5.99)$ & $(6.35)$ & $(4.89)$ \\
MSE & {$[40.57]$} & {$[67.55]$} & {$[379.75]$} & {$[466.25]$} & {$[235.08]$} \\
\hline
\end{tabular}

Note: The table shows the mean and standard deviation of each estimator. The mean square error for the models with 20 proxies are given in the last line. See text for details. 


\section{Table 2: Measuring the Effect of Wealth on Children's School Attendance In India}

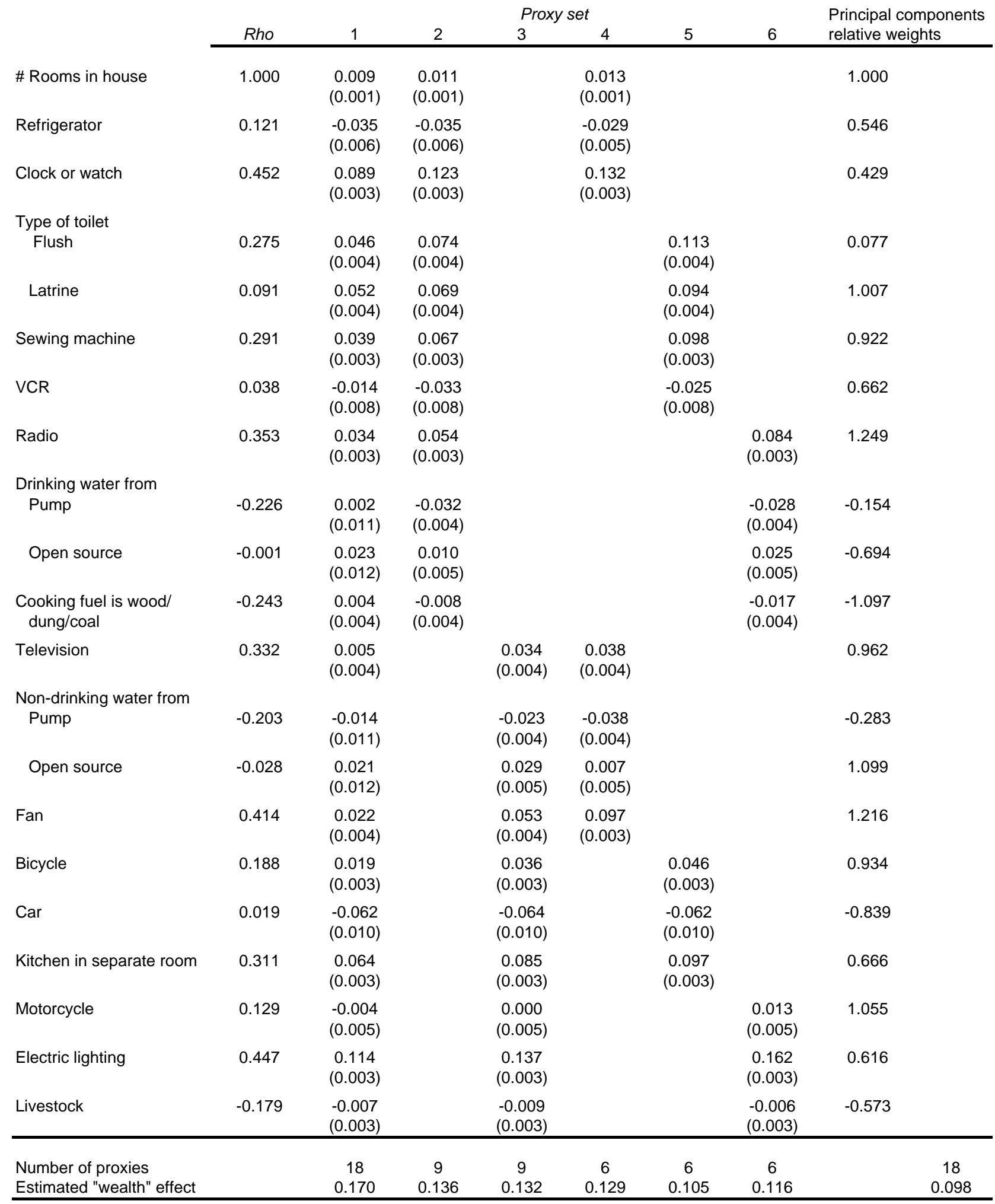

Note: Data is from the Demographic and Health Survey of India. Sample size is 109,973 . The dependent variable is an indicator that the child is enrolled in school. The model also controls for the child's sex and age, the head of household's sex, age and education, and the log family size. 
Figure 1: The Effect of Family Income on Children's Reading Comprehension Score

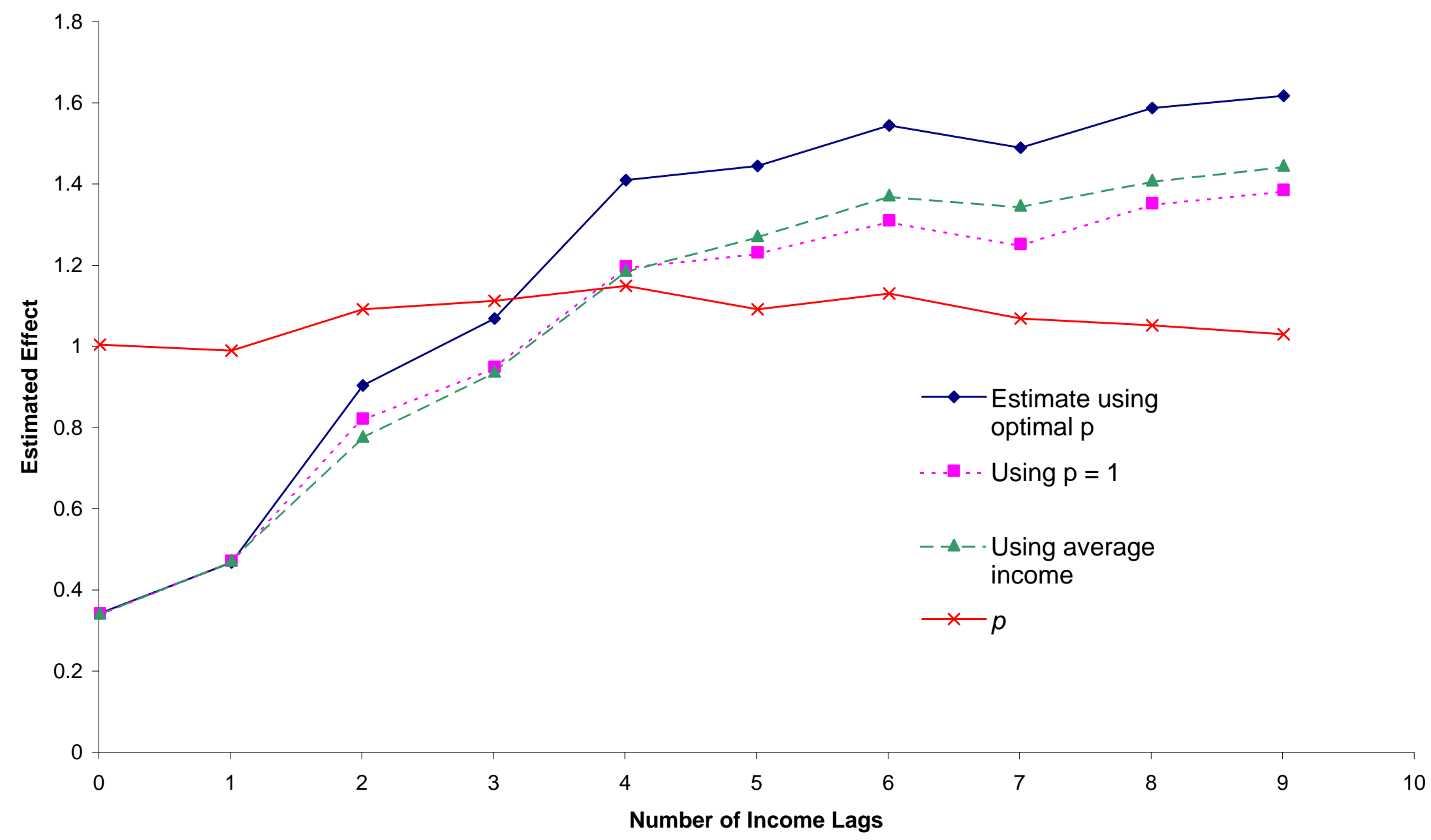

Note: Data is from the NLSY-Children, 1979-1998. All models also include controls for the log family size, the child's sex, age, and race, the mother's age and education, whether the mother's spouse is present, and if so, his age and education, year effects, and the mother's AFQT score. 